\title{
Formas de inclusión-exclusión en el sistema educativo chileno: el movimiento estudiantil secundario 2006-2011*
}

\author{
Elia Gutiérrez Martínez' (DD \\ Raúl Zamorano Farías" (D)
}

\section{RESUMEN}

En este trabajo se analiza la forma en la que opera el sistema educativo chileno (SECL), desde la Reforma de 1980 (impuesta por la dictadura militar y que continúa orientando la organización del sistema educativo), y el surgimiento del movimiento estudiantil secundario (MES). Cabe señalar que tal acercamiento no se observa como fruto de una relación causal ni como producto derivado de una determinada "crisis" social, sino más bien como un proceso social que se fue generando a lo largo del tiempo y que derivó en la significativa movilización estudiantil a comienzos del siglo XXI en Chile. Con ese objetivo se utilizan dos conceptos de la teoría general de los sistemas sociales de Niklas Luhmann, para observar cómo en el operar de todo sistema social se producen procesos de inclusión/exclusión y específicamente, analizar las formas en que se estabilizan estos fenómenos en la sociedad.

\section{PALABRAS CLAVE}

sistema educativo; educación mercantil; inclusión/exclusión; igualdad/desigualdad.

*Este artículo tiene su origen en la tesis doctoral de Elia Gutiérrez Martínez: El movimiento estudiantil secundario en Chile, 2006-2011: continuidades y cambios (2017).

'Universidad Intercontinental, Ciudad de México, México.

"Universidad Nacional Autónoma de México, Ciudad de México, México. 


\title{
FORMS OF INCLUSION-EXCLUSION
}

\section{IN THE CHILEAN EDUCATIONAL SYSTEM:}

\section{THE SECONDARY STUDENT MOVEMENT 2006-2011}

\begin{abstract}
This paper will analyze the forms of operation of the Chilean Educational System (SECL) operates, since the 1980 Reform (imposed by the military dictatorship and which continues to orient specifically the organization of the Educational System), and the consequent development of the named Secondary Student Movement (MES). It should be noted that such an approach is not seen as the result of a causal relationship or as a product of a given social "crisis", but rather as a social process that was generated over time and led to significant student mobilization at the beginning of the 21st century in Chile. With this objective, two concepts of Niklas Luhmann General Theory of Social Systems are used to observe how every social system inclusion/exclusion processes take place in the operation and, specifically, to analyze the ways in which these phenomena are stabilized in society.
\end{abstract}

KEYWORDS

educational system; mercantile education; inclusion/exclusion; equality/inequality.

\section{FORMAS DE INCLUSÃO-EXCLUSÃO NO SISTEMA EDUCATIVO CHILENO: MOVIMENTO ESTUDANTIL SECUNDÁRIO 2006-2011}

RESUMO

Este artigo descreve a maneira pela qual o sistema da educação chileno (SECL) opera, desde a reforma de 1980 (imposta pela ditadura militar e que continua a orientar a organização do sistema da educação), e o surgimento do movimento estudantil secundário (MES). Deve-se notar que tal abordagem não é vista como o resultado de uma relação causal ou como um subproduto de uma determinada "crise" social, mas sim como um processo social gerado ao longo do tempo, resultando em uma mobilização estudantil significativa no início do século XXI, no Chile. Com esse objetivo, dois conceitos da teoria geral dos sistemas sociais de Niklas Luhmann são usados para observar o funcionamento de todos os processos de inclusão/exclusão de todo o sistema social e, especificamente, as maneiras que estabilizam estes fenômenos na sociedade.

PALAVRAS-CHAVE

sistema de educação; educação mercantil; inclusão/exclusão; igualdade/desigualdade. 


\section{INTRODUCCIÓN}

A partir del golpe de Estado contra el gobierno de Salvador Allende (septiembre de 1973) y con la aprobación de la Constitución de $1980,{ }^{1}$ en Chile se va configurando un modelo educativo ad hoc al nuevo esquema político-económico de la dictadura, el cual se estabiliza y profundiza con el retorno de los gobiernos democráticamente electos $(1990)^{2}$ y actualmente sigue incluso vigente. Esta estructura institucional implementada por la dictadura - en concreto en el sistema educativo chileno (SECL) -, está basada en un esquema de mercado, la cual ha permanecido sin cambios significativos de fondo (Bellei y Pérez, 2010, p. 20). ${ }^{3}$

Bajo tales condiciones, en el sistema educativo en Chile se generaron nuevas formas de diferenciación y de inclusión/exclusión que modificaron las prácticas y expectativas de los ciudadanos y derivaron en un creciente malestar social y estudiantil, el cual se fue acumulando a lo largo de varios años. Precisamente, serán los estudiantes de nivel secundario quienes comenzaron a organizarse políticamente a principios del nuevo siglo, sobre todo en el año 2006, para cuestionar el modelo educativo bajo el cual se habían formado y los efectos que este produjo. Los estudiantes se organizan para problematizar políticamente aquello que no había sido tematizado como problema en el sistema educativo, ${ }^{4}$ instituyendo nuevos sentidos, expectativas y orientaciones para protestar y movilizarse.

Para los estudiantes la educación en Chile era la constatación de una situación de "injusticia" y "agravio" (en el sentido jurídico del concepto), siendo este el marco referencial que orientó la movilización como una posibilidad, un sentido por el cual valía la pena luchar. Al cuestionar algunos aspectos del SECL, tales como el lucro en la educación y el consecuente incremento de segregación social, los estudiantes enmarcan y definen a la educación como un derecho que deviene como un bien de consumo (mercancía), vulnerado porque agravia un derecho social fundamental

1 La Constitución de 1980 estableció mecanismos como verdaderos cerrojos constitucionales al implementar medidas que impiden cambios sustantivos en el modelo político (Cumplido, 2006).

2 Los cuales para paliar las diferencias que se establecieron al interior del sistema educativo, implementaron diversas medidas en pos de "mejorar" la educación, tales como la renovación del instrumental pedagógico y curricular con que opera la educación escolar y la mejora del equipamiento en los liceos.

3 Luego de 33 años, fue recién en el año 2016, con base en las protestas estudiantiles (2006 y 2011), que se presentaron iniciativas de reforma en la educación durante el segundo mandato de Michelle Bachelet.

4 Quienes elaboran definiciones de los problemas y al hacerlo los "enmarcan". Lo anterior no significa que los problemas no existan, pero pueden pasar desapercibidos. Por lo tanto, la movilización social (MS) o protesta social (PS) (en la semántica de Luhmann), funcionan como dispositivos de resonancia en el sistema social (Snow y Benford, 2000; Luhmann, 2010). 
(reconocido constitucionalmente) que, en los hechos, no ha sido garantizado por el Estado. ${ }^{5}$

\section{INCLUSIÓN/EXCLUSIÓN, IGUALDAD/ DESIGUALDAD EN EL SISTEMA EDUCATIVO}

Es común que en cierta parte de la literatura sociológica y pedagógica se analicen de modo separado y de confrontación los conceptos inclusión y exclusión en torno al tema educativo, también es frecuente que estos conceptos sean observados con un sesgo valorativo o moral (deber ser), que apela a la igualdad - contra la desigualdad - solo que sin definir qué es lo que se está indicando y además, sin observar que "toda" igualdad produce desigualdad. Por ejemplo, la inclusión puede ser vista como sinónimo de "justicia en la educación", la cual debe seguir los intereses de los menos favorecidos (Connel, 1997, p. 25), ${ }^{6}$ mientras que la exclusión es entendida - comúnmente - como "aquello que está fuera del sistema". Tal que "la exclusión social implica una cierta imagen dual de la sociedad, en la que existe un sector "integrado" y otro "excluido" (Tezanos, 1999, p. 12). Desde esta perspectiva, los excluidos no participan en lo social, lo cual es "injusto y malo".

Desde la aportación de la Teoría General de Sistemas Sociales, Luhmann señala que "nadie está fuera del sistema" porque la sociedad misma es autorreferencial, lo que en ella sucede no puede estar fuera de su acontecer. La sociedad se autoobserva y es constreñida a reflexionar su operación y sus consecuencias.

5 Reconociendo que la relación entre el surgimiento del MES y la forma en la que opera el SECL es evidente, tal relación no fue ni es lineal o única. Al respecto, in lato sensu teóricamente un movimiento social es resultado de un proceso social amplio, en el que los actores vinculados se organizan, crean sentidos e identidades, construyen el problema, el cual se define constantemente a través de las acciones colectivas (Della Porta y Diani, 2011). Desde esta perspectiva, los movimientos sociales son más bien producto de una inversión organizativa de los actores involucrados (Melucci, 2010, p. 12-14). En esta dirección Snow y Benford señalan que "los marcos de acción colectiva funcionan como 'dispositivos' que subrayan o adornan la gravedad y la 'injusticia' de una situación social o redefinen como injusto o inmoral lo que previamente era considerado desafortunado, aunque tal vez tolerable" (Snow y Benford, 2000, p. 137). Para Luhmann, la protesta social es el reflejo de la sociedad funcionalmente diferenciada - el espejo de la sociedad moderna - ya que toda operación de inclusión genera su lado de la diferencia; exclusión. Es decir, toda inclusión presupone formas la exclusión contra la cual se protesta, se vuelve a protestar y así recursivamente (Luhmann, 1996).

6 Otro ejemplo es la semántica que se produce en los documentos, análisis o proyectos políticos en torno al tema educativo generado por los organismos internacionales, en los cuales se pretende eliminar la exclusión y lograr la inclusión educativa total por medio de compromisos políticos y planeaciones estratégicas. Al respecto, el compromiso que se estableció por la UNESCO (2000-2015) en Dakar Senegal, donde los países participantes establecieron como meta común lograr: la educación de calidad para todos. Los resultados de tal política se observaron quince años después como desafíos y promesas no cumplidas en las que se presentaron nuevas formas de diferenciación en la educación, así como de procesos de exclusión e inclusión, sobre todo para la región latinoamericana y africana. Véase OREALC, 2013. 
Parte de esa reflexión es en torno al fenómeno de la exclusión que ella misma genera y que re-genera - paradojalmente - con las tentativas de limitarlo o eliminarlo (Luhmann, 2007, p. 492).

Si bien en el sistema educativo y sobre la base de las políticas educativas se busca la inclusión total (educación para todos) y con ello se pretende eliminar la exclusión de modo definitivo, las diferencias que la sociedad produce no pueden eliminarse bajo principios políticos, jurídicos o morales (los cuales terminan solo en buenas intenciones para la retórica política); y aún cuando sí es posible la disminución de sus consecuencias, esto no evita efectos colaterales, no contemplados. Es decir, no evita nuevas diferencias y formas de exclusión en la inclusión. Dicho de otra forma, el sistema educativo establece su función entre educar/no educar, en donde no es excluido quien repruebe, sino quien no figure para el sistema como comunicación, quien "no exista” para este. Además, al ser los procesos de inclusión/ exclusión y los de igualdad/desigualdad regulados por una sociedad funcionalmente diferenciada, implica que se pierde la posibilidad de que la inclusión o la igualdad sean totalmente controladas por algún principio unitario.

El devenir evolutivo del proceso de diferenciación de la sociedad trae como resultado que en la sociedad se generen conflictos, demandas, exigencias y expectativas que no siempre se cumplen y que producen nuevas exigencias y expectativas en función de esas prácticas (Luhmann, 2007). Así para Luhmann, en la construcción y operatividad del orden social la inclusión es "la cara interna de una forma, cuya cara externa es la exclusión”, por ello solo es pertinente hablar de inclusión si está presente la exclusión y viceversa. La inclusión considera desde un contexto comunicativo a los seres humanos al tomarlos en cuenta, "se trata del modo y manera en que los seres humanos son tratados como personas”(Luhmann, 1998, p. 172).

Deviene importante señalar que para la teoría general de los sistemas sociales, las personas son equivalentes a comunicaciones, ${ }^{7}$ y no deben observarse como individuos o reducirse a masa biológica, toda vez que la masa biológica constituye el presupuesto del ser humano. Si la sociedad se autorreproduce por medio de comunicaciones, las personas que se incluyen en dicho proceso no son propiamente hombres o mujeres que ingresan o egresan a ella, sino que son entornos de comunicación (psíquicos). Desde esta perspectiva teórica, cuando se habla de la inclusión de personas se hace referencia a un conjunto de expectativas a los cuales el sistema atribuye características individuales (Luhmann, 2007).

"La inclusión se entiende entonces como la capacidad de participar en la vida social. La exclusión por el contrario no permite la visibilidad de las personas, por lo que se interrumpe la comunicación, lo cual impide la formación de expectativas complejas, recíprocas y reflexivas" (Corsi, 1998, p. 31); pero nadie está totalmente incluido o excluido de las comunicaciones sociales, a menos que sea reducido solo a masa biológica (Luhmann, 1998, 2007).

Como se ha señalado, los sistemas sociales generan formas de inclusión y de exclusión de modo inevitable al reproducirse a sí mismos. No obstante, los sis-

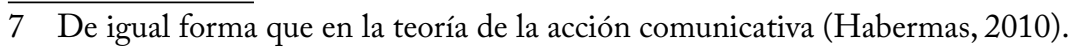


temas pueden seguir operando con las diferencias y exclusiones que ellos mismos producen (Luhmann, 2007). También en el sistema educativo (SE), se producen diversas formas de inclusión/exclusión, de igualdad/desigualdad toda vez que en su operar diferenciado, el sistema educativo se "auto-observa" y con ello señala y describe los fenómenos que se producen en su interior; mientras que los fenómenos de exclusión o desigualdad social, requieren ser justificados y solucionados por el sistema de la política (los gobiernos de turno). La inclusión y la igualdad en cambio no requieren justificación, sino solo su organización (Luhmann, 1998; Corsi, 1998).

Por lo tanto, es imprescindible observar que en cualquier contexto social se generan nuevas y viejas formas operativas de inclusión/exclusión social; así como de igualdad/desigualdad. ${ }^{8}$ La cuestión es observar entonces, cómo se crean estos fenómenos en lo específico de cada contexto social y cuáles son las estructuras sociales disponibles para que estas sean posibles, se reproduzcan y estabilicen, como presupuestos de la variación para producir nuevas selecciones, cambios y también inclusiones y exclusiones (Luhmann, 2007).

\section{EL SISTEMA EDUCATIVO EN CHILE}

A partir de la implementación de la Constitución de 1980, en Chile se fue configurando un modelo educativo con base en las reglas del mercado. El principio de libertad de enseñanza (LE), fue uno de los postulados que se retoman ideológicamente del pasado y que calzaron perfecto en el modelo educativo mercantil. Si bien la LE surgió con la Reforma Constitucional de 1874, en medio de pugnas entre liberales y conservadores por la dirección que debía tomar la educación, ${ }^{9}$ un siglo después y tras el golpe militar, la libertad de enseñanza fue y será entendida como: (1) la posibilidad de los padres de elegir la educación para sus hijos; y (2) la libertad de los particulares en crear y administrar colegios, y en donde es el deber del Estado financiarlos y apoyarlos "sin condiciones", en igualdad de trato frente a la educación pública.

Bajo esta égida, se descentralizó el sistema educativo y se reorganizó la municipalización de la educación básica — primaria y secundaria —, en la cual operó la transferencia desde una administración central (Ministerio Nacional de Educación) a una administración local del sistema educativo bajo la responsabilidad de los municipios, lo que desde la política mercantil tenía como fin activar la competencia a

8 Bajo esta premisa no tiene sentido de hablar de "crisis" en la sociedad, como fundamentos "causales" de los MS o de protesta.

9 Por un lado, los liberales, afirmaban que era el Estado quien debía mantener, irrenunciablemente, la dirección de las escuelas y liceos, con el fin de proteger los principios de la República por sobre los intereses evangelizadores de la Iglesia Católica. Por su parte, la Iglesia Católica reclamaba que no podía existir una formación en libertad si existía un monopolio en la contratación de docentes, en la rendición de exámenes, en las estructuras curriculares y en los métodos de enseñanza. La LE busca garantizar la posibilidad de que existan proyectos educativos alternativos a la educación provista por el Estado. Idea que calzó perfectamente en la Constitución de 1980 (Jackson, 2014). 
nivel municipal. Entre otros efectos, en la práctica y desde el primer momento esta medida agudizó y profundizó las diferencias de los servicios educativos, que ofrecen los municipios según y sobre la base de su capacidad económica. ${ }^{10}$

A tales efectos se creó entonces la subvención estatal por medio del bono educativo, solo que en términos de subvención, el Estado no distinguió a los colegios públicos de privados, tal que la "igualdad de trato" suponía el mismo aporte tanto a los colegios con fines de lucro (privados con subvención) cuanto a los públicos municipalizados (sin fines de lucro). La competencia entre los colegios se instituye, entonces, sobre la base de desiguales condiciones (con o sin fin de lucro), y será potenciada por los procesos de selección que establecen las escuelas respecto a los alumnos: siendo los colegios subvencionados quienes seleccionan a sus estudiantes bajo criterios arbitrarios, amparados en las pruebas de conocimientos, la capacidad económica de las familias, su status social e incluso el credo religioso del usuario (Mena y Corbalán, 2010); lo que derivó en fenómenos como la segregación social y la ghetización escolar. ${ }^{11}$

Esencialmente, desde su origen y en lógica político-económica (1980), se buscaba entonces estructurar un sistema educativo con una administración descentralizada (operada por los municipios) que en teoría tuviese mayores capacidades de administración económica tanto a nivel comunal cuanto a nivel escuela (Bellei, 2003, p. 3). Será una década después (1990), que esta lógica - orientada por las directrices neoliberales - se cristaliza a través de la Ley Orgánica Constitucional de la Educación (LOCE), como uno más de los legados del régimen militar, el cual entró en vigor justamente el último día del mandato de Augusto Pinochet. Es decir, fue paradójicamente implementado por el gobierno democráticamente electo por la ciudadanía (Concertación de partidos por la democracia),${ }^{12}$ estabilizando estructuras y procesos políticos autoritarios en un contexto supuestamente "democrático".

Al respecto Santa Cruz y Olmedo (2012), señalan que esto se debió a la fragilidad institucional de la transición hacia la democracia en la cual el gobierno de la Concertación tomó el poder, toda vez que este fue un pacto entre las fuerzas políticas y militares. Así, para Brunner (1990, p. 6), la constitucionalidad chilena se ha movido entre pactos. Uno de estos fue que el nuevo gobierno de la concertación reconoció que no se podría desarrollar una lucha contra el régimen al margen de la

10 Es claro que si los municipios administran el sistema educativo, lo hacen sobre la base de la recaudación de impuestos y del aporte del Estado. Por lo tanto, lo que recauda un municipio de la zona económicamente alta ("Las Condes") es significativamente distinto a lo que recaudan los municipios ubicados en las zonas marginales ("E1 Bosque").

11 Se entiende por segregación social a la "desigual distribución que poseen los diversos grupos sociales, ya sea entre unidades de organización diferentes, entre zonas geográficas o en una combinación de ambas, tales diferencias de distribución afectan las probabilidades de interacción entre miembros de los diferentes grupos sociales" (Bellei, 2013, p. 329). En la práctica, el resultado es que prevalezca la homogeneidad en la composición de los alumnos en las escuelas (ghetización educativa) (Redondo et al., 2004).

12 La Concertación fue una coalición de partidos de centro-izquierda, la cual se oponía a la dictadura de Augusto Pinochet, a quien derrotó electoralmente en el plebiscito Nacional el 5 de octubre de 1988. 
institucionalidad surgida de este. "Desde ese instante quedó descartada cualquier alternativa opositora más radical, de ruptura y confrontación directa con el régimen militar".

Con la Constitución de 1980 se implementa entonces un modelo educativo cuyo fin era introducir una lógica de mercado en la educación. Sin embargo, este plan no logró establecerse plenamente, por contradicciones político-ideológicas al interior del propio régimen y por la soterrada disputa entre una visión estatista y otra neoliberal. Esto generó un modelo educativo "mixto", en donde se plasmará de forma sui generis el debate entre ambas visiones (Almonacid et al., 2008, p. 10). El resultado fue $y$ ha sido un sistema educativo administrado por el Estado y, por otro, de impulso y fuerte influencia del mercado en la educación. Ejemplo de ello es que el Ministerio de Educación se reserva el derecho al proyecto y manejo curricular, así como al reconocimiento oficial de los establecimientos educativos, tanto públicos cuanto privados, los cuales deben ceñirse a los contenidos curriculares nacionales, solo que los planes y programas no son obligatorios para todos los colegios. ${ }^{13}$

De esta forma, la prevalencia del Estado en los contenidos curriculares de la educación chilena, pese a la fuerte influencia de la política mercantil, obedece a que el poder militar logró imponer y conservar ciertos intereses de tipo ideológico, en donde "el propósito fundamental era mantener esa estructura descentralizada y la identidad nacional" (Almonacid et al., 2008, p. 11-12). ${ }^{14}$

Como resultado, el funcionamiento al interior del SECL se perfiló sobre la base de una lógica estatista, amparada en el pretérito ideal del Estado Docente ${ }^{15}$ "un esquema de ingeniería social que presuponía dos imperativos: crear una suerte de "religión civil" o "cultura cívica" que construiría y orientaría a la unidad nacional (Nef Novella, 2000, p. 3) y, por otra, el Estado Subsidiario cuyos fines se orientaban por políticas de mercado con prevalencia a la paulatina privatización del sistema educativo; una amalgama irreconciliable entre dos posturas, la estatista y la mercantil (Ruiz Schneider, 2012; Núñez,2011). Es precisamente en la política del subsidio en

13 Se debe hacer una distinción entre Marco curricular y Planes y Programas de Estudio. Mientras el Marco curricular es obligatorio en Chile para todo el sistema escolar, tanto de administración pública cuanto privada, los planes y programas no son obligatorios para todos los colegios.

14 Entrevista a Iván Núñez (Almonacid et al., 2008), asesor del Ministro de Educación desde el año de 1990 hasta el año 2008.

15 La construcción del Estado Docente se inició con la promulgación de la Ley General de Instrucción Primaria en 1860. Esta ley estableció la obligación del Estado de convertirse en el principal sostenedor de la educación y la gratuidad escolar en su etapa inicial. "El Estado Docente significó inclusión social creciente y fortalecimiento y expansión de la educación pública, pero también implicó la clausura o la pérdida de espacio de muchas formas de diversidad y de los impulsos que desde la base del sistema y de los movimientos sociales presionaban por un mayor protagonismo e incidencia en la definición y despliegue de las políticas educativas del país, desde una lógica de autonomía de lo social de lo político-estatal"(Águila, 2013, p. 3). Posteriormente, con la dictadura y la interrupción del sistema democrático, durante los años ochenta, se observa al Estado Docente como obsoleto y se señala como "necesario" que sea sustituido por un tipo de Estado Subsidiario. 
donde se expresa mejor la fusión de esta ideología católica - conservadora militar con la fusión del paradigma económico neoliberal, adoptado e implementado en la educación chilena.

El sistema de financiamiento educativo vía subvención por alumno atendido, se orientó entonces sobre la base de la competencia, que llevaría a la "calidad" entre las escuelas públicas (municipales) y las privadas con subvención, las cuales, pese a sus diferencias intrínsecas, se disputan el atraer a las familias y con ello la obtención de recursos públicos por medio del sistema de voucher, acrecentando y estabilizando la lógica de la ganancia económica (mercancía), así como la expansión de la educación por parte de las instituciones privadas con fines de lucro, y en menor medida, sin fines de lucro; ${ }^{16}$ y también un sistema de evaluación nacional que mide la "calidad" 17 de los establecimientos escolares y de ése modo crea las condiciones para que los padres de familia puedan elegir "el mejor" colegio para sus hijos (Bellei y Vanni, 2015).

Tal que la "vuelta" a la democracia trajo aparejado el rediseño de la política y del sistema educativo, orientando la implementación de programas para el mejoramiento y la equidad de la educación, cuya facticidad operativa tenía por fundamento una mayor inversión y estrategias para restablecer el papel activo del Estado. Sin embargo, esto no tuvo el impacto necesario ni esperado, más allá de las limitaciones tanto en su diseño como en su puesta en práctica, toda vez que

Su efectividad fue limitada porque operaban dentro de contextos institucionales que eran incapaces de modificar y que eran a menudo una fuerza en contra de los cambios requeridos. (Bellei y Vanni, 2015, p. 6)

Es precisamente en este tipo de intervenciones con base en políticas cuyos fines se basan en lograr mayor igualdad o inclusión, en las que se puede observar cómo el sistema al poner en operación sus funciones genera nuevos (y en ocasiones) más complicados esquemas de desigualdad y exclusión, incluso de aquellos que busca remediar, sobre todo cuando este esquema educativo ha generado un modelo que funciona como un cuasi mercado. Es decir, como un sistema que establece la diferencia entre el comprador, el proveedor y un elemento de elección del usuario entre distintos proveedores (Laval, 2004). ${ }^{18}$

Es necesario señalar que el SECL, se divide - de modo esquemático en tres subsistemas.Entre ellos figuran la educación privada sin subvención (de élite), la cual representa el 7\% de la población escolarizada; la educación privada subven-

16 Bellei y Pérez (2010, p. 19), señalan que en 1980 un 75\% de los jóvenes estudiaban en liceos públicos, mientras que en 1986 estos habían caído a 58\%, en 1995, a 52\% y en 2007, a 43\%.

17 Sin especificar nunca qué significa eso de calidad: ¿Como fórmula de contingencia de qué, en qué y cómo?

18 Otra característica del cuasi mercado ha sido la "particular" combinación de los elementos administrativos, financieros y pedagógicos (Whitty, 2000 citado por Almonacid et al., 2008, p. 5). 
cionada, la cual es financiada de manera compartida entre el Estado y los padres de familia, concentrando un $43 \%$ de la población escolarizada. Por su parte,

el sector público, financiado totalmente por el sistema de voucher administrado por el municipio - que a comienzos de los años 1980 concentraba un 75,3\% de la matrícula escolar - se ha visto reducido a poco menos de un $50 \%$ del alumnado a nivel nacional, el cual para el año 2011 retenía únicamente un $42 \%$ de la población en edad escolar, proveniente en su gran mayoría de los sectores más pobres de la población urbana, rural e indígena. (Concha, 2011, p. 1)

Consecuentemente, en los colegios subvencionados con fines de lucro las cifras han ido en aumento.

En este esquema el Estado funge como administrador de los recursos; aunque también define los objetivos del sistema educativo (programas y contenidos de enseñanza y evaluación de los aprendizajes); la normativa (funcionamiento y financiamiento), y las condiciones (estatuto docente e infraestructura), de modo que el sector público emula sus mecanismos de operación como lo haría el mercado.

En sus primeros momentos (década de los 1990), algunas de las medidas correctivas al "modelo" estuvieron basadas en criterios de "austeridad" y "eficiencia", ya que los costes (gastos para la producción de bienes y servicios) que suponía la expansión educativa eran enormes, tal que el medio fundamental para lograrlo será a través de la creación del subsidio, el cual funciona con base en una política diferencial, en la que el Estado ya no provee de modo total los recursos, sino a través de una intervención parcial del financiamiento, consolidando así el esquema de cofinanciamiento en donde el Estado aportaría una parte y los beneficiarios otra.

El esquema de cofinanciamiento debe entonces diferenciar a los alumnos y sus familias según su capacidad de pago por los servicios educativos que estos reciben: quienes tienen ingresos altos deben pagar el costo real, los de ingreso medio deberían reembolsar una parte, y a los pobres corresponde entregarles gratuitamente la atención. (Cornia, 1987 citado en Franco, 1997, p. 46)

Con ello termina por cristalizarse la preeminencia de las lógicas mercantiles del proceso educativo y también activa, de forma casi delirante, la participación de la iniciativa privada en la educación, sobre la base del postulado de la "libertad de enseñanza”. Así el sistema crea las estructuras sociales disponibles (jurídicas, económicas y hasta culturales) y otorga las garantías para que cualquiera pueda invertir en ella, ${ }^{19}$ y en donde el financiamiento de los colegios depende entonces del tipo de cada establecimiento. Mientras los colegios particulares pagados se financian con el cobro de arancel sin ayuda del Estado, los establecimientos municipales y privados subvencionados son en general "gratuitos" y reciben principalmente aportes fiscales (Almonacid et al., 2008), aunque en el caso de estos últimos las familias realizan un aporte extra (copago).

19 Amparado en la figura que se denomina "sostenedor educativo" (dueños de los colegios) y que tiene el estatus de "cooperador de la función educativa del Estado". 
Como se ha indicado, el mecanismo que el Estado implementó para la subvención de los colegios municipales y privados, fue vía el voucher educativo.

El voucher o bono educativo fue un modelo de administración económica ideado por Milton Friedman a mediados del siglo pasado (1955). Tal Modelo fue adoptado y desarrollado con características propias en el SECL (1981) como un mecanismo de subvención del Estado, sobre la base de un modelo de financiamiento en torno a la demanda de las familias. Entre los argumentos a favor de este sistema están: (1) el derecho a la elección del consumidor; (2) el desarrollo personal como responsabilidad de las personas; (3) la competencia que se establece entre las escuelas públicas/privadas; y (4) la "igualdad" de oportunidades como consecuencia de los otros tres principios (Cosse, 2000, p. 161-164).

$\mathrm{El}$ voucher se asigna a los alumnos bajo un criterio de eficiencia y constancia, es decir, pago por alumno atendido (por cada día de asistencia al colegio). Según la propia legislación chilena, el Estado tiene la obligación de financiar a los colegios privados con subvención y municipales, a través de aquellos usuarios que lo soliciten. En este modelo los colegios reciben recursos en función del número de estudiantes matriculados o por asistencia efectiva.

Si bien la política del voucher tuvo en principio una base homogénea; es decir, un mismo valor de subsidio para todos los estudiantes, esta lógica política - basada en un principio de igualdad - no consideró que las diferencias de origen no desaparecen, por el contrario, terminan instaurando nuevas. Sin embargo, según lo esperado por la política educativa, este modelo derivaría en un sistema eficiente, basado en la competencia entre los colegios por la obtención de recursos y, con ello, el logro de la 'calidad' educativa esperada. Esto generaría que los alumnos no abandonasen los colegios, ya que las escuelas, al requerir de su financiamiento, harían lo posible por atraer y retener a sus estudiantes. Cuestión que contribuiría además al incremento en la cobertura (Prieto, citado por Almonacid et al., 2008, p. 13). ${ }^{20}$

Será en el año 2008 que con la política del voucher se intente operar sobre la base de la equidad y del reconocer las diferencias de origen, implementando el subsidio diferenciado: el Subsidio de Educación Preferencial (SEP). Esta "nueva" lógica reconoce "que educar niños y niñas en contextos de mayor vulnerabilidad socioeconómica es más complejo y más caro y se asume entonces que el Estado debe poner más recursos ahí donde más se necesita”(Raczynski et al., 2013, p. 166).

Señalan los autores que después de siete años de implementado el SEP, "los desafíos de esta política son tanto o más grandes que sus primeros resultados" (Raczynski et al., 2013, p. 186), ya que y como se ha indicado, cualquier política compensatoria busca beneficiar por un lado a aquellos a quienes va dirigida, sin embargo, no elimina las diferencias que surgen de este proceso (incluye y excluye al mismo tiempo); toda vez que las diferencias que surgen no son una constancia de sumas, en la cual al aumentar de un lado se disminuye el otro. Por el contrario,

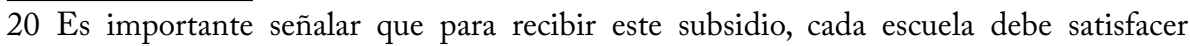
ciertos requerimientos establecidos por la autoridad. Bajo esta operatividad, se parte del supuesto que los colegios que no tienen alumnos suficientes para cubrir sus costos dejan de operar y salen del sistema (Gallego y Sapelli, 2007, p. 265-266). 
estas derivarán en nuevos e inesperados efectos secundarios (Corsi, 1998). Al respecto, es importante considerar que incluso en los sistemas educativos que funcionan con base en una lógica mercantil, el proceso de exclusión no ocurre a priori, toda vez que para la operatividad de este y de cualquier otro sistema social, se requieren de la inclusión y la igualdad, solo que como presupuestos básicos no determinados.

La política de subvención si bien parte de esta lógica, incluyendo a quienes educa, en la práctica define y determina diferencias intrínsecas entre ambos tipos de colegios toda vez que sirve a los fines del cuasi mercado educativo reproduciendo y profundizando esas diferencias en las instituciones educativas, las cuales al realizar su función generan de modo inevitable una exclusión selectiva, toda vez que el esquema de mercado funciona por medio de un oferente (colegios) y un demandante (alumnos), donde la competencia se establece como condición fundamental del sistema, y aún cuando el Estado es el garante de ese derecho, nunca debe sustituir al mercado educativo, salvo en el caso de que estas organizaciones (escuelas) se vean imposibilitadas para realizar sus fines por cuenta propia (Ruiz Schneider, 2010).

\section{LAS ESTRUCTURAS POLÍTICAS AL INTERIOR DEL SECL}

Las estructuras político-jurídicas (Constitución) y la organización del sistema educativo que han prevalecido en Chile desde el gobierno militar (1973-1990), y que permanecen hasta hoy en día, han forjado las condiciones operativas y las estructuras que hacen posible observar y explicar cómo se han generado los efectos de inclusión-exclusión, de igualdad-desigualdad en el SECL (recordemos que los sistemas sociales al operar, generan procesos de inclusión/exclusión; igualdad/ desigualdad en tanto forma como unidad de la diferencia, en donde lo sustantivo es observar precisamente el cómo se produce este fenómeno).

En el plano empírico esto se puede observar en la política del subsidio a los colegios públicos y subvencionados por medio del voucher educativo, como un principio de inclusión e igualdad dentro de una lógica de administración mercantil, sin embargo, dicha política partió de establecer un principio de igualdad en la desigualdad, es decir, un mismo subsidio a colegios con fines de lucro - subvencionados - y sin fines de lucro - públicos - , lo que en la práctica derivó en nuevas y más profundas formas de desigualdad y exclusión social.

Orientado por las directrices que definió la Constitución de 1980, el SECL introdujo y consolidó un tipo de política basada en la "libre empresa" (apertura de colegios privados que competían en desiguales condiciones con colegios públicos), sobre la base del derecho de la "libertad de elección" de los usuarios. En teoría esta idea establece un principio de inclusión e igualdad al ser los padres de familia (apoderados) quienes pueden elegir el colegio que más les convenga, solo que en la práctica tal 'elección' está definida y determinada en función de la capacidad económica de las familias, por lo que el principio de elección no es tal, ya que las familias con recursos limitados no pueden pagar un aporte extra, por lo tanto no pueden elegir, sino solo conformarse con la "educación" gratuita (la cual también es de diversa calidad, siendo los colegios emblemáticos los de mejores resultados escolares). Esto da como resultado la paradójica relación de la exclusión en la inclusión. 
En otras palabras, la desregulación del "negocio" educativo y las laxas condiciones - amparadas normativamente - que tienen los particulares para abrir una escuela, son elementos que han alentado a que este tipo de estructura permanezca, se reproduzca y a su vez derive en la paulatina minimización y pauperización de la educación pública. Fenómeno que evidentemente vulnera el derecho de que los ciudadanos reciban una educación pública más allá de los criterios mercantiles, toda vez que aquellos que no pueden pagar o aportar para la educación de sus hijos no pueden ejercer el supuesto "derecho" a esa de libre elección. Ergo, la política de la "libertad de enseñanza" es demagógica, selectiva y excluyente, además de ser una ficción, toda vez que la capacidad de selección está determinada y recae en los colegios de copago, no así en los usuarios.

Si los colegios subvencionados seleccionan a sus alumnos sobre la base del criterio mercantil (introduciendo nuevas y más profundas formas de exclusión), excluyendo a aquellos estudiantes que no reúnan las condiciones que establecen los dueños de las escuelas (promedio escolar, capacidad de pago y credo religioso, en el caso de los colegios católicos), en la práctica son ellos los que eligen a los "mejores" alumnos, aplicando criterios arbitrarios que profundizan la ghetización del sistema educativo y representan en algunos casos dramáticas formas de exclusión escolar y social. Eso que Carnoy y McEwan (1997) denominan como el "descreme" educativo.

Sin embargo, este es un proceso en el cual también las propias familias contribuyen de modo indirecto, al preferir "ser elegidas" por los colegios de mayor prestigio (avalando esta nueva forma de ghetización y exclusión en la inclusión), pues para ellas está implícito el valor de status (roce social), de las familias con las que interactuarán sus hijos y no tanto la "calidad" del colegio (Canales et al., 2016, p. 96-98), tal que la 'calidad' también viene entendida y definida por criterios mercantiles antes que por criterios de formación. De forma paralela, las familias de bajos recursos se orientan no por el prestigio o la calidad del colegio, si no por la cercanía de la escuela con los hogares (ligado a un factor económico) o, por principios de asistencia social (control disciplinario) (Espínola, 1989; Gallego y Sapelli, 2007; Joiko, 2012).

Este doble proceso de selección - colegios y familias -, en los hechos reproduce y estabiliza la inclusión y la exclusión y se traduce en nuevas formas de segmentación y desigualdad social. De modo que y como resultado de lo anterior, se re-introducen nuevas formas de selección, discriminación, autoexclusión e inclusión al conformarse grupos sociales homogéneos al interior de las escuelas, donde la clase social y el status ligado a ella, tienen un peso importante, estabilizando los procesos de ghetización educativa que, paralelamente, van estabilizando, "naturalizando" y determinado estas formas segregación escolar y social.

$\mathrm{Si}$ a lo anterior se agrega que la determinante económica se establece precisamente en la cúspide de la política de la descentralización escolar o municipalización, y supone ser un medio para "mejorar" y hacer eficiente la administración de los recursos, en la practica la municipalización ha derivado en una recaudación diferenciada entre los colegios, de modo que los municipios con mayor renta tienen más captación de recursos que los de menores ingresos, profundizando y determinado una vez más las evidentes diferencias entre las escuelas, incluso entre las mismas escuelas públicas. 
Estos ejemplos en conjunto dan cuenta del paradójico fenómeno de la inclusión en la exclusión y de exclusión en la inclusión o de procesos de desigualdad en la igualdad al interior del SECL. Cuestiones que constituyeron un cúmulo de malestar y de expectativas no cumplidas que pueden (más no necesariamente) generar conflictos y el surgimiento de la protesta social.

\section{EL SURGIMIENTO DEL MES}

En el caso que aquí ocupa, fueron los estudiantes de nivel medio o secundario, quienes de cara a este estado de cosas comenzaron a organizarse y manifestarse políticamente desde el año 2001 ("el mochilazo"), articulando la movilización estudiantil que tenía como objetivo - en un primer momento - la gratuidad en el pasaje escolar. Será hasta el año 2006 cuando se presentan manifestaciones de protesta más organizadas y con un mayor conocimiento de las problemáticas en torno al operar del sistema educativo y que afectaban directamente a los estudiantes. Empero, esto fue producto de un largo proceso que se fue articulando paulatinamente con este actor colectivo en concreto y no así el resultado directo de una crisis particular en el sistema educativo. ${ }^{21}$

Bajo esta premisa es posible considerar entonces que el 'surgimiento' del MES no es el resultado lineal y único de las consecuencias que se desprenden del SECL, si no una posibilidad entre otras. ${ }^{22}$ En otras palabras, es claro que operativamente los MS y la protesta requieren primeramente un colectivo organizado que dé coherencia y articulación a eso que el movimiento atribuye como un "problema", lo cual es una construcción constante que se enmarca en una red de sentidos, identidades y significados que los actores sociales generan al organizarse y que no está per se definido.

Si bien los estudiantes secundarios y las organizaciones estudiantiles (Asamblea Coordinadora de Estudiantes Secundarios, ACES y posteriormente la Coordinadora Nacional de Estudiantes Secundarios CONES) que participaron en los movimientos del año 2006 y del año 2011, han sido un actor social que se ha ido constituyendo a lo largo del tiempo, ${ }^{23}$ éstos lograron presencia significativa a comienzos del nuevo siglo, aún cuando:

21 Es necesario considerar que en la sociedad siempre existe el conflicto, porque las expectativas sociales no siempre son cumplidas, pero esto no deriva necesariamente en protestas y/o movilizaciones sociales, sobre todo cuando en la sociedad moderna se puede siempre protestar por todo y sobre todo.

22 Se ha entrecomillado el término surgimiento, pues se parte del supuesto teórico que los Movimientos sociales no son acontecimientos repentinos en la historia, sino procesos sociales o inversiones organizativas (Melucci, 2010; Tarrow, 2004).

23 Es importante señalar que los estudiantes secundarios como actor social ha participado de modo discontinuo a lo largo del siglo XX: será a mediados del siglo XX que se agrupan principalmente en la Federación de Estudiantes Secundarios (FESES). Sin embargo, es a comienzos del siglo XXI cuando su organización sufre cambios sustantivos, se disuelve y surge la ACES, cuya principal característica es su relativa autonomía de los partidos políticos, la horizontalidad de su organización, la mayor participación de sus bases y, sobre todo, el cambio en sus intereses de lucha cuya orientación parte de su realidad específica y no tanto de los intereses de los partidos políticos (Donoso, 2014). 
'el movimiento de los secundarios' (como se los denomina generalmente) ha sido más débil, menos estructurado, más dependiente de otros sujetos sociales [...]. Sin embargo, en determinadas coyunturas, la situación ha sido muy distinta, adquiriendo una notable fuerza y protagonismo. (Rojas Flores, 2009, p. 472)

El movimiento estudiantil secundario (MES), surgió en el año 2006, y mantuvo un ciclo de movilizaciones breves (de abril a junio), y tuvo como demandas principales la gratuidad del pase escolar (PE: tarjeta de descuento para el transporte público), la gratuidad de la Prueba de Selección Universitaria para los tres primeros quintiles de la población (PSU: examen obligatorio para todos los estudiantes del país en su último año de estudios secundarios, este debe ser pagado por el estudiante), la revisión de la Jornada Escolar Completa (JEC: programa que tiene por objetivo lograr la calidad educativa a través de una mayor carga académica), ${ }^{24}$ y los débiles resultados académicos en los establecimientos que implementaron el programa (Torres, 2010, p. 12). Demandas que luego serán conocidas como "de corto plazo".

Conforme se fue profundizando el conflicto, las demandas y expectativas de los estudiantes derivaron en otras de mayor alcance, las cuales para ser posibles requieren cambios sustantivos a nivel de política educativa e incluso del orden Constitucional. Entre las más relevantes destacan:

1. la desmunicipalización de los colegios públicos, de modo centralizado a través del Ministerio de Educación;

2. la derogación de la Ley Orgánica Constitucional de Enseñanza (LOCE); y

3. la educación gratuita, pública y de excelencia.

Como se indicó, sobre la base la Constitución de 1980 y la LGE, se legaliza un modelo educativo basado en los principios del mercado y el cambio en el rol rector del Estado a otro de tipo subsidiario o administrador de los recursos, con base al esquema de la ley de la oferta y la demanda, lo cual precisamente será cuestionado por los estudiantes, orientando los reclamos que el MES 2006 pone a debate, problematizando políticamente aquello que no era considerado como un problema educativo para el sistema político: la educación pública, gratuita y de excelencia.

En el MES 2006 participaron dos mil establecimientos de educación entre liceos públicos y privados. Prácticamente un millón de estudiantes se movilizaron en forma activa, sobrepasando en cantidad a las movilizaciones en contra de la dictadura de Pinochet. Recordemos que tras dos meses de movilización y una serie de marchas, tomas de liceos y paros de diverso tipo, y luego de muchos desaciertos de las autoridades, a finales de marzo del año 2006, la entonces recién electa presidenta Michelle Bachelet (2006-2010), minimizó el incipiente conflicto estudiantil, actitud que lejos de menguarlo lo profundizó (Torres, 2010, p. 11).

Ante las protestas estudiantiles, el Ejecutivo en turno anunció que transformaría "el problema en oportunidad", para establecer un diálogo abierto con los

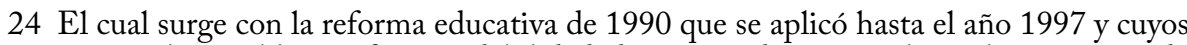
principales problemas fueron: debilidad de contenidos curriculares, limitaciones de recursos humanos y materiales (García-Huidobro y Concha, 2009, p. 7). 
estudiantes (Pinochet, 2007, p. 3) ${ }^{25}$ Posteriormente y bajo una serie de rechazos a los ofrecimientos del gobierno, el Ejecutivo convocó esta vez a expertos y a un reducido número de estudiantes (12 de un total de 78 participantes) para constituir el llamado Consejo Asesor para la Calidad de la Educación, del cual surgió la derogación de la LOCE y su sustitución por la Ley General de Educación (LGE), así como la creación de una Superintendencia de Educación, cuyo fin era velar por el correcto funcionamiento de los establecimientos privados subvencionados por el Estado y obligaba a las sociedades con fines de lucro que administraban colegios, a transformarse en corporaciones privadas sin fines de lucro. Sin embargo, esta última enmienda fue rechazada tanto por los dueños de los colegios subvencionados (sostenedores) como por algunos miembros del gobierno con intereses concretos, para que esos cambios no se llevaran a cabo (Donoso, 2014). ${ }^{26}$

La LGE fue aprobada en el año 2007 y puesta en marcha dos años después en 2009, y si bien hubo algunos cambios a partir de esta nueva ley, dichos cambios fueron más de forma que de fondo al "perpetuarse el lucro en la educación subvencionada con recursos del Estado y la dependencia de los colegios públicos a las municipalidades que seguían igual de desfinanciadas que antes” (Gerter y Ramos, 2011, p. 8). En ese contexto, parte de las demandas que surgen del MES 2006 consiguieron ser tematizadas en el sistema político (la derogación de la LOCE y su sustitución por la LGE), pero estas no lograron cambios de fondo; solo sirvieron para dar continuidad a la política de educación mercantil que sigue vigente en Chile.

Posteriormente, en el año 2011, estalla un nuevo ciclo de movilizaciones, lideradas - en primera instancia - por los estudiantes universitarios organizados en la Confederación de Estudiantes de Chile $(\mathrm{CONFECH})^{27}$ quienes protestaron en contra del alto costo de la educación superior y por el retraso en el otorgamiento de becas (educación superior que en Chile es totalmente privada). A estas movilizaciones se sumaron los estudiantes secundarios, quienes participaron con sus propias organizaciones estudiantiles: la Asamblea Coordinadora de Estudiantes Secundarios (ACES) y posteriormente la Coordinadora Nacional de Estudiantes Secundarios (CONES) (que surge ya iniciado el conflicto estudiantil). Estos colectivos que tenían diferencias en sus formas de organización, en su tendencia política y en

25 Como consecuencia de la lenta reacción que mostró el gobierno en sus inicios ante el conflicto estudiantil, la presidenta Bachelet realiza el primer cambio de gabinete a 125 días de emprender su gestión, el Ministro de Educación Martin Zilic fue reemplazado en su cargo por Yasna Provoste (Simonsen, 2011).

26 "Luego de seis meses en que el proyecto fue discutido en la Comisión de Educación del Congreso, las dos coaliciones con representación parlamentaria, la Concertación (Coalición de la que surge el Gobierno de M. Bachelet) y Alianza por Chile (Coalición de derecha), sellaron un acuerdo 'histórico' sobre un proyecto definitivo en noviembre de 2007, el cual privilegia el derecho al lucro de los sostenedores de colegios subvencionados" (Gerter y Ramos, 2011, p. 8).

27 La CONFECH responde a la rearticulación de la organización estudiantil universitaria en el contexto de la dictadura militar y fue creada durante el I Congreso Nacional de Estudiantes Universitarios realizado en el mes de octubre de 1984, en la ciudad de Valparaíso. 
algunas de sus demandas, también compartían objetivos en común. Por ejemplo, que el Estado debe cumplir un rol protagónico en la educación y no solo ser un administrador de los recursos y que la educación en todos los niveles educativos debiera orientarse con base en un modelo público y gratuito.

Tanto el MES2006 como el ME2011 fueron movimientos con una proyección mediática importante, los estudiantes supieron desplegar a su favor el uso de las redes de la información y la comunicación, tanto las clásicas (prensa, televisión) como de las nuevas tecnologías (redes sociales), acentuando los signos del descontento estudiantil y el cuestionamiento al modelo educativo en su conjunto, así como las demandas en pos de reformas profundas al interior del sistema educativo: derogación de la LGE que rige actualmente la educación en Chile.

Como forma de apaciguar las demandas estudiantiles y la prolongación de las manifestaciones de diversos actores sociales ${ }^{28}$ el gobierno de Sebastián Piñera (2011-2015) promovió un paquete de reformas y planes educativos que llevaron el polémico nombre de "Revolución Educativa". Entre las ofertas del mandatario destacaron el "Gran Acuerdo Nacional de la Educación" (GANE) y el "Fondo por la Educación" (FE; cuyo monto fue de 4.000 millones de dólares). Mas, a las declaraciones del Presidente Piñera los estudiantes universitarios y secundarios respondieron con fuertes críticas, movilizaciones y tomas de colegios, pues para ellos tales medidas no estaban en "sintonía con sus demandas", toda vez que se continuaba con los esquemas mercantiles que hasta entonces habían operado en el sistema educativo.

$\mathrm{Al}$ poner en el centro de la discusión el tema de la educación pública, gratuita y de excelencia en todos los niveles educativos, los estudiantes universitarios y secundarios instalan y enmarcan el tema de la gratuidad como el principal "agravio" (perjuicio o no aplicación de un derecho) por el que vale la pena luchar. Para los estudiantes era claro que el Estado debería fortalecerse para poder redirigir la educación bajo otros referentes

Debemos pasar del reinado autoritario del principio de subsidio al mercado [educativo], al principio de universalidad [en la educación] garantizada por el Estado. (Boric, 2012, p. 34)

Así, el tema que se instala como un continuum y transversal en el movimiento (2006-2011) fue esencialmente el de la gratuidad de la educación en todos los niveles.

Hacia finales del año 2011 y después de un evidente desgaste de los disidentes, la represión policial, los desacuerdos entre el gobierno y los estudiantes, las amenazas de sanciones como la cancelación de la matrícula y la presión ante la eminente pérdida del año escolar, así como las propias pugnas al interior de las organizaciones estudiantiles, los estudiantes decidieron deponer las tomas de sus colegios, pero conscientes de que "no habían perdido un año escolar, sino que [eso] había sido una inversión” (Arrué, 2012, p. 4).

28 Participaron distintas organizaciones y actores sociales (Colegio de Profesores, sindicatos de trabajadores, padres de familia e incluso algunos dueños de colegios subvencionados y rectores de universidades "tradicionales"). 
Al final el saldo del movimiento fue de un alto costo interno y, paralelamente, la autopercepción de haber logrado pocos triunfos o resultados concretos, lo cual provocó un 'repliegue' momentáneo en las organizaciones estudiantiles. ${ }^{29}$ Empero, los cambios más visibles del movimiento fueron principalmente simbólicos (Castro, 2013), pues se logró cuestionar al modelo educativo en conjunto y profundizar y extender a otros sistemas sociales el conflicto, toda vez que se potenciaron las formas de tematizar políticamente y problematizar las lógicas y consecuencias mercantiles que orientan el SECL.

Con todos sus bemoles, es evidente que la movilización estudiantil fue capaz de recrear sentido e identidad, en pos de ese objetivo. Hubo un "impacto simbólico y cultural" el cual transformó en parte un sistema de creencias e ideologías, estableciendo nuevas identidades y marcos de acción (Castro, 2013, p.11).Marcos de acción que se enfrentan a otros - incluso más poderosos - que se han apropiado, definido y controlado la institucionalidad vigente, en este caso bajo la figura de los grupos políticos y empresariales fuertemente vinculados al negocio educativo, abriendo además la puerta para cuestionar estas premisas no solo sobre el tema educativo en el ámbito del sistema educativo sino también en el sistema político-jurídico.

La lucha de los estudiantes secundarios fue un movimiento que buscó no solo cambiar las reglas de operación del SECL. Fue además una forma en la que los actores lograron re-incluirse en un proceso político del que históricamente las organizaciones estudiantiles en Chile han participado desde principios del siglo XX, tematizando y politizando la creación de un proyecto educativo "propio" y de demandas de corto y largo plazo (independientemente de que este fuese considerado o no por los políticos en turno), generando así otras formas de inclusión en la exclusión.

\section{A MODO DE CONCLUSIÓN}

Un movimiento social nunca es una verdad, es solo una posibilidad (entre muchas otras)...

Nexus

Las estructuras socio-jurídicas disponibles que han repercutido en la reproducción del sistema mercantil en la educación en Chile siguen un curso legal que requiere de cambios profundos a nivel político y jurídico, para poder configurarse como cambios efectivos al interior del sistema educativo. En tal sentido, los estudiantes lograron justamente tematizar en el sistema de la política algunas de sus demandas que, sin embargo, este re-interpreta y dispone de acuerdo a sus propias pautas operativas, lo cual puede observarse, por ejemplo, con la fanfarroneada

29 "En aquel momento, si no hubiéramos tenido la experiencia del 2006, habríamos dicho 'tratemos de lograr algo' [...]. Antes, la idea siempre era que cada año la Federación tenía que avanzar en un tópico particular; siempre tratábamos de lograr algo después de una movilización. Las protestas del 2011 fueron fuertemente criticadas a este respecto. La gente decía que no se había logrado nada" (Hunneus, presidente de la FECH en el 2011, entrevistado por Donoso, 2014, p. 39). 
derogación de la LOCE y la creación de la LGE como un sustituto funcional, que en la práctica mantiene la institucionalidad asentada desde la época de la dictadura.

Es por ello que cualquier cambio que se implemente en la política respecto a las demandas estudiantiles queda en la "interpretación" si estos permanecen en entredicho, porque en la práctica continúan operando sobre la base de estructuras sociales normativas que estabilizan un tipo de educación mercantil y, que de modo inevitable, introducen nuevas diferencias que generarán también nuevas expectativas, demandas y con ello la posibilidad de la instauración de saldos pendientes y nuevos conflictos. De modo que, e independientemente de los triunfos tangibles y simbólicos respecto a las demandas del MES, su papel ha sido el de ser un "eco", una "resonancia" que problematiza y subraya una parte de la realidad - que se presenta como intolerable - y por lo tanto se vislumbra también como un posible y necesario cambio en la sociedad.

El papel que jugó entonces el movimiento estudiantil fue el de poner en evidencia y problematizar en el sistema de la política — la mercantilización educativa - un problema que no era considerado como tal. Es decir, subrayar, remarcar, problematizar y crear orgánicamente un marco de referencia que le diera articulación y proyección. La organización política estudiantil evidenció lo que se había estabilizado normativamente en el terreno de la política (y de las leyes como máximo ejemplo de recursividad histórica), mostrando que las protestas sociales representan esas fuerzas que al politizar los conflictos y agravios 'empujan' al permanente cambio en la sociedad desde la sociedad. ${ }^{30}$

ElMES fue posible porque los estudiantes no eran un grupo excluido del sistema educativo, sino precisamente y porque estaban incluidos pudieron confrontarlo, analizarlo y politizarlo desde sus propias experiencias de lucha, y si bien - en los hechos - algunos de los estudiantes son excluidos a través de diversos mecanismos que se producen al interior del sistema educativo, vía paradójicas mixturas de inclusión en la exclusión o de exclusión en la inclusión, tales contradicciones orientaron su movilización, la cual apela a la inclusión a partir de sus propuestas y demandas en torno a la educación y en una mayor participación política de lo social.

Los estudiantes lucharon por ser re-incluidos en la sociedad a través de una educación de excelencia, pero no determinada en la lógica definida por el mercado, sino abierta por medio de la auto-inclusión a través de su participación política, para así apelar a espacios de democratización social. Tal que el movimiento estudiantil ha sido y es una forma de inclusión, que con sus propios medios y particularidades

30 Para algunos teóricos sociales reconocer la movilización social implica reconocer una cierta capacidad salvadora y emancipadora desde fuera de la sociedad (¿?), pues y según estas mesiánicas visiones, la marginalidad de quienes se manifiestan estaría a la base de las disrupciones colectivas (reventones históricos) de carácter radical y violento, evidencia factual del cumplimiento de su misión histórica. Sin embargo, con demasiada frecuencia se habla del "proyecto" de un movimiento cuando lo que se capta es únicamente su ideología (o la ideología del teórico que "(de) codifica”). Hay que reaccionar contra una confusión de este orden y subrayar que este término no debe introducir, en ningún caso, un análisis del sentido vivido del movimiento, reducido a la construcción necesariamente artificial de sus principios, emociones y valores (Zamorano Farías, 2001). 
generacionales e identitarias, se organiza desde lo político como alternativa a la institucionalidad vigente (la política, con la cual no se sienten plenamente identificados), la cual también enmarca los límites y posibilidades a los cuales ellos se enfrentan.

\section{REFERENCIAS}

Águila, E. Del Estado docente al Estado subsidiario: de la escuela pública selectiva a la escuela segregada. In: CÁTEDRA FOUCAULT, 2013, Santiago de Chile, Departamento de Estudios Pedagógicos, Universidad de Chile, 2013.

Almonacid, C.; Luzón, A. Torres, M. Cuasi mercado educacional en Chile: el discurso de los tomadores de decisión. Education Policy Analysis Archives/Archivos Analiticos de Políticas Educativas, v. 16, n. 8, p. 1-47, 2008.

Arrué, M. El movimiento estudiantil en Chile (2011-2012): Una lucha contra la discriminación. Amérique Latine Histoire et Mémoire. Francia: Les Cabiers ALHIM, n. 24, 2012. Disponible en: <http://alhim.revues.org/4388>. Acceso en: ago. 2015.

Bellei, C. Veinte años de políticas en educación media en Chile: 1980-2000: de la autoregulación del mercado al compromiso público. In: CARIola, L.; Bellei, C.; Nuñez, I. Veinte años de politicas de educación media en Chile. París: Instituto Internacional de Planeamiento de la Educación, 2003. p. 173-266.

. El estudio de la segregación socioeconómica y académica de la educación chilena, Estudios Pedagógicos, v. 39, n. 1, p.325-345, 2013. Disponible en: <http://www. redalyc.org/articulo.oa?id=173528596018>. Acceso en: mar. 2015.

.; PÉrez V. Conocer más para vivir mejor. Educación y conocimiento en Chile en la perspectiva del Bicentenario. In: Lagos, R. (Ed.). Cien años de luces y sombras. Santiago de Chile: Taurus, 2010. p. 1-77.

.; VAnni, X. The evolution of educational policy in Chile, 1980-2014. In: Schwartzman, S. (Ed.). Education in South America. London: Bloomsbury Publishing, 2015. p. 179-200.

Boric, G. Horizontes del movimiento estudiantil In: Vallejo, C.; González, E.; Fuentes, I.; Huenchunao, J.; Boric, G.; Titelman, N.; Gálvez, R.; Sarabia, C.; Díaz, D.; Cuello Araya, M. Transformar la sociedad haciendo camino. Santiago de Chile: Editorial Aún Creemos en los Sueños, 2012. p. 31-36.

Brunner, J. Chile: claves de una transición pactada. Nueva Sociedad, Buenos Aires, n. 106, p. 6-12, mar./abr. 1990.

Canales, M.; Bellei, C.; Orellana, V. ¿Por qué elegir una escuela privada subvencionada?: sectores medios emergentes y elección de escuela en un sistema de mercado. Estudios Pedagógicos, Valdivia, v. 42, n. 3, p. 89-109, 2016.

Carnoy, M.; McEwan P. Public Investments of Private Schools? A reconstruction of Educational Improvements in Chile. Palo Alto: Standford University Press, 1997.

Castro, F. Movimiento estudiantil chileno 2011-2013: impactos y consecuencias. 2013. $46 \mathrm{f}$. Tesis (Licenciatura en Ciencia Política) - Pontificia Universidad Católica de Chile, Santiago, Chile, 2013. 
Concha, C. Chile: cerrando el ciclo de la administración educativa municipal. In: Seminario Regional Descentralización de la Educación en Centroamérica. Mejora de La Eficiencia y la Participación, 2011, GIZ, ICAP y Guatemala: Ministerio de Educación de Guatemala. 26-28 jul., 2011.

Connell, R. W. Escuelas y justicia social. Madrid: Ediciones Morata, 1997.

Corsi, G. Redes de la exclusión In: Castañeda, F. S.; Vázquez, A. C. (Coords.). Redes de Inclusión:la construcción social de la autoridad. Ciudad de México: MAPorrúa,1998.p.29-43.

Cosse, G. El sistema del voucher educativo: ¿una nueva panacea para América Latina? Revista Uruguaya de Ciencia Politica, Montevideo, v. 12, p. 157-178, 2000.

Cumplido, F. La constitución chilena de 1980 y sus efectos en el régimen político. Anuario de Derecho Constitucional Latinoamericano. México: Instituto de Investigaciones Jurídicas, UNAM, 2006. p. 105-113. Disponible en: <https://revistascolaboracion.juridicas.unam.mx/index.php/anuario-derecho-constitucional/article/ view/30294/27344>. Acceso en: 10 oct. 2018.

Della Porta, D.; Diani, M. Los movimientos sociales. España: Centro de Investigaciones Sociológicas (CIS); Editorial Computlense, 2011. (Colección Debate Social).

Donoso, S. La reconstrucción de la acción colectiva en el Chile post-transición: el caso del movimiento estudiantil. Buenos Aires: CLACSO, 2014. p. 1-45. (Documento de trabajo). Disponible en: <http://movimientoestudiantil.cl/wp-content/ uploads/2015/12/110-La-reconstrucci\%C2\%A2n-de-la-acci\%C2\%A2n-colectiva-enel-Chile-post-transici\%C2\%A2n-el-caso-del-movimiento-estudiantil-Sofia-Donoso. pdf $>$. Acceso en: 28 ago. 2016.

Espínola, V. Los resultados del modelo económico en la enseñanza básica: la demanda tiene la palabra. In: García-Huidobro, J. E. (Ed.). Escuela, calidad e igualdad: los desafíos para educar en democracia. Santiago de Chile: CIDE, 1989. p. 41-81.

Franco, R. Paradigmas de la política social en América Latina. In: Larín, R. M.; Kruijt, D.; van Vucht Tijssen, L. (Eds.). Pobreza, exclusión y politica social San José: FLACSO Sede Costa Rica, 1997. p. 35-58.

Gallego, F.; Sapelli, C. El financiamiento de la educación en Chile: una evaluación. Pensamiento Educativo. Revista de Investigación Educacional Latinoamericana, v. 40, n. 1, p. 263-284, 2007.

García-Huidobro, J. E.; Concha, C. Jornada escolar completa: la experiencia chilena. Chile: Centro de Estudios en Políticas y Prácticas en Educación (CEPPE), 2009. Disponible en: <https://www.academia.edu/35225291/Jornada_escolar_completa_la_ experiencia_chilena>. Acceso en: 2 feb. 2016.

Gerter,D.; Ramos, C. Movimiento Estudiantilen Chile: una respuesta a la carencia de Estado. Valdivia:Universidad Austral de Chile,2011.p.1-11.Disponible en: <https://elmoderador.files. wordpress.com/2011/06/movimiento-estudiantil-en-chile-20061.pdf>. Acceso en:2 feb.2016. Gutiérrez Martínez, E. El movimiento estudiantil secundario en Chile, 2006-2011: Continuidades y cambios. 2017. 225f. Tesis (Doctorado en Pedagogía) - Universidad Nacional Autónoma de México, México. Disponible en: <http://bibliotecacentral.unam. mx/tesis.html>. Acceso en: 25 abr. 2017. 
Habermas, J. Teoría de la acción comunicativa Tomo I. Racionalidad de la acción y racionalización social. Tomo II. Crítica de la razón funcionalista. Madrid: Trotta, 2010.

Jackson, G. Los nudos de la reforma educacional. Chile: Centro de Investigación Periodística, 2014. Disponible en: <https://www.lidereseducativos.cl/los-nudos-de-lareforma-educacional-explicados-en-8-puntos/>. Acceso en: 11 sep. 2017.

Jогко, S. El cuasi-mercado educativo en Chile: desarrollo y consecuencias. Revista Electrónica Diálogos Educativos, v. 12, n. 23, p. 148-174, 2012. Disponible en: <https:// www.academia.edu/4632433/E1_cuasimercado_educativo_en_Chile_Desarrollo_y_ consecuencias>. Acceso en: 09 jul. 2017.

Laval, C. La escuela no es una empresa: el ataque neoliberal a la enseñanza pública. Barcelona: Paidós, 2004.

Luhmann, N. Protest. Systemtheorie und soziale Bewegungen. Frankfurt am Main: Suhrkamp Verlag, 1996.

. Complejidad y modernidad: de la unidad a la diferencia. Madrid: Trotta, 1998. . La sociedad de la sociedad. México: Herder; Universidad Iberoamericana, 2007. . Organización y decisión. México: Herder; Universidad Iberoamericana, 2010. Melucci,A.Acción colectiva, vida cotidiana y democracia. México: El Colegio de México, 2010. Mena,P.; Corbalán P.La selección escolar: una cuestión de libertad, competencia,igualdad e integración. In: Bellei, C.; Contreras, D.; Valenzuela J.P.(Eds.). Ecos de la revolución pingüina: avances, debates y silencios en la reforma educacional. Santiago de Chile: Fondo de las Naciones Unidas para la Infancia UNICEF; Universidad de Chile, 2010. p. 331-363. Nef Novella, J. El Concepto de Estado Subsidiario y la Educación como bien de mercado: Un bosquejo de análisis político. Revista Enfoques Educacionales. v. 2, n. 2, 2000. Disponible en: <https://enfoqueseducacionales.uchile.cl/index.php/REE/article/ view/47047>. Acceso en: 03 jun. 2016.

NúñEz, S. Principio de subsidiariedad y mercado educacional In: Coloquio Educación y Mercado, Universidad de Chile, Santiago, 5-7 oct., 2011.

Oficina Regional de Educación para América Latina y el Caribe (OREALC). Situación Educativa de América Latina y el Caribe: hacia la educación de calidad para todos al 2015. Santiago: OREALC/UNESCO, 2013.

Pinochet, S. La "Revolución Pingüina" y el cambio cultural en Chile. Argentina: CLACSO; Biblioteca Virtual, 2007. p. 1-29. Disponible en:

<http://bibliotecavirtual.clacso.org.ar/ar/libros/becas/2007/cultura/silva.pdf >. Acceso en: 5 mayo 2016.

Raczynski,D.; MuÑoz,G.; Weinstein,J.; Pascual,J. Subvención escolar preferencial(SEP) en Chile: un intento por equilibrar la macro y micro política escolar. Revista Iberoamericana sobre Calidad, Efcacia y Cambio en Educación, Madrid, v. 11, n. 2, p. 164-193, 2013.

Redondo, J.; Descouvières, C.; Rojas, K. Equidad y calidad de la educación en Chile. Reflexiones e investigaciones de eficiencia de la educación obligatoria (1990-2001). Santiago de Chile: Universidad de Chile; LOM, 2004. 
Rojas Flores, J. Los estudiantes secundarios durante la Unidad Popular, 1970-1973. Historia (Santiago), v. 42, n. 2, p. 471-503, dic. 2009.

Ruiz Schneider, C. De la República al mercado. Ideas educacionales y políticas en Chile. Santiago de Chile: LOM, 2010.

. Lo público y lo privado en la educación chilena. Santiago de Chile: Facultad de Filosofía y Humanidades, Universidad de Chile, 2012. (Documento de trabajo).

Santa Cruz, E.; Olmedo, A. Neoliberalismo y creación de 'sentido común': crisis educativa y medios de comunicación en Chile, Revista de Curriculum y formación del profesorado, Granada, v. 16, n. 3, p. 167-190, 2012.

Simonsen, E. Mala educación: historia de la revolución escolar. Santiago de Chile: Ed. Debate, 2011.

Snow, D.; Benford, R. Master frames and cycles of protest. In: Morris, A.; Mcclurg, C.; Mueller, E. Frontiers in social movements theory. New Haven, Londres: Yale University Press, 2000. p. 133-155.

TARrow, S. El poder en movimiento: los movimientos sociales, la acción política. Madrid: Alianza Editorial, 2004.

Tezanos, J. F. Tendencias en desigualdad y exclusión social. Tercer foro sobre tendencias sociales. Madrid: Editorial Sistema, 1999.

TORREs, R. Juventud, resistencia y cambio social: el movimiento de estudiantes secundarios como un actor político en la sociedad chilena post-Pinochet (1986-2006). In: Axe XI, Symposium 40. Independencias-Dependencias-Interdependencias, VI Congreso CEISAL 2010, Jun. 2010, Toulouse, 2010. Disponible en: <https://halshs.archives-ouvertes.fr/ halshs-00498869/>. Acceso en: mayo 2016.

ZAMORANo FARías, R. Entre la teoría y la acción: dilemas sobre la acción colectiva popular. Santiago de Chile: 1988-1992. México: Juan Pablos Editor, 2001.

\section{SOBRE LOS AUTORES}

Elia Gutiérrez Martínez es doctora en Pedagogía por la Universidad Nacional Autónoma de México (México). Profesora de la Universidad Intercontinental (México).

E-mail:eliza74_zapa@yahoo.com.mx

Raúl Zamorano Farías es doctor en Filosofía Jurídica por la Università degli Studi di Lecce (Italia). Profesor de la Universidad Nacional Autónoma de México (México).

E-mail: rzamorano61@gmail.com

Recibido el 17 de abril de 2017

Aprobado el 8 de diciembre de 2017

(c) 2018 Associação Nacional de Pós-Graduação e Pesquisa em Educação - ANPEd Este es un artículo de acceso abierto distribuido en términos de licencia Creative Commons. 